This item was submitted to Loughborough's Research Repository by the author.

Items in Figshare are protected by copyright, with all rights reserved, unless otherwise indicated.

\title{
Lateral edge effects on heat/mass transfer on a finite width surface within a turbulent boundary layer
}

PLEASE CITE THE PUBLISHED VERSION

https://doi.org/10.1016/j.ijheatmasstransfer.2019.04.016

PUBLISHER

(C) Elsevier

VERSION

AM (Accepted Manuscript)

\section{PUBLISHER STATEMENT}

This paper was accepted for publication in the journal International Journal of Heat and Mass Transfer and the definitive published version is available at https://doi.org/10.1016/j.ijheatmasstransfer.2019.04.016.

\section{LICENCE}

CC BY-NC-ND 4.0

\section{REPOSITORY RECORD}

Angelino, Matteo, Richard J. Goldstein, and Fabio Gori. 2019. "Lateral Edge Effects on Heat/mass Transfer on a Finite Width Surface Within a Turbulent Boundary Layer". figshare. https://hdl.handle.net/2134/37615. 


\title{
Lateral Edge Effects on Heat/Mass Transfer on a Finite Width Surface within a Turbulent
}

\section{Boundary Layer}

\author{
M. Angelino ${ }^{a^{*}}$, R. J. Goldstein ${ }^{b}$, F. Gori ${ }^{c}$ \\ ${ }^{\text {a }}$ Department of Aeronautical and Automotive Engineering, Loughborough University, \\ Loughborough, LE11 3TU, UK. \\ b Regents' and James J. Ryan Professor of Mechanical Engineering, Turbulent Convective Heat Transfer \\ Laboratory, Department of Mechanical Engineering, University of Minnesota, Minneapolis, MN 55113, USA. \\ ${ }^{c}$ Laboratory of Thermo-Fluid Dynamics, Department of Industrial Engineering, \\ University of Rome "Tor Vergata”, 00133 Rome, Italy. \\ "Corresponding author: m.angelino@lboro.ac.uk
}

\begin{abstract}
Numerical simulations of the local heat/mass transfer on a finite width surface within a turbulent boundary layer are presented. Different approaches to the RANS modelling of the turbulent heat/mass flux are compared to Large Eddy Simulations (LES). Mass transfer experiments conducted with the naphthalene sublimation technique are used as validation. The isotropic eddy viscosity model, Simple Gradient Diffusion Hypothesis (SGDH), is shown to underestimate the span-wise effects. Its anisotropic extension, Generalized Gradient Diffusion Hypothesis (GGDH), improves the prediction, but still does not account for near-wall contribution in strongly dissimilar velocity and temperature/concentration fields, even in combination with a wall-sensitive second-moment closure model such as the Elliptic Blending Reynolds Stress Model (EB-RSM). A more complete turbulent heat flux model based on the elliptic blending approach, the Elliptic Blending GGDH (EB-GGDH) presents very good agreement with LES and with the experiments, confirming the need for more advanced turbulent heat flux modelling in applications with strong three-dimensional effects.
\end{abstract}

Keywords: Turbulent boundary layer; Heat/Mass Transfer Analogy; Large-Eddy Simulation (LES); Elliptic Blending Reynolds Stress Model (EB-RSM); Turbulent heat transfer model (EB-GGDH).

\section{NOMENCLATURE}

$\begin{array}{ll}J & \text { scalar flux vector } \\ k & \text { turbulent kinetic energy } \\ L & \text { turbulent length scale } \\ \mathrm{Nu} & \text { Nusselt number } \\ p & \text { pressure } \\ \mathrm{Pr} & \text { Prandtl number } \\ \mathrm{Pr}_{\mathrm{t}} & \text { turbulent Prandtl number } \\ R_{i j} & \text { Reynolds stresses } \\ \mathrm{Re} & \text { Reynolds number } \\ \mathrm{Sc} & \text { Schmidt number } \\ \mathrm{Sh} & \text { Sherwood number }\end{array}$


Stanton number

$\mathrm{St}_{\mathrm{C}}$

centerline Stanton number

$S$

strain rate

$T$

turbulent time scale

$u_{i}$

instantaneous velocity vector

$\overline{u_{i}^{\prime} u_{j}^{\prime}}$

Reynolds stress tensor

$\overline{u^{\prime} u^{\prime}}$

stream-wise normal stress

$\overline{u^{\prime} v^{\prime}}$

shear stress

$\overline{u_{j}^{\prime} \theta^{\prime}}$

turbulent heat/mass flux vector

$U$

mean stream-wise velocity

$\overline{v^{\prime} v^{\prime}}$

vertical normal stress

$\overline{v_{j}^{\prime} \theta^{\prime}}$

wall-normal turbulent heat flux

$\overline{w^{\prime} w^{\prime}}$

span-wise normal stress

$\overline{w_{j}^{\prime} \theta^{\prime}}$

$x$

span-wise turbulent heat flux

stream-wise distance from the plate leading edge

$x^{\prime}$

stream-wise distance from virtual origin of the turbulent boundary layer

y

vertical distance from the wall

$Z$

span-wise distance from the lateral edge of the plate

\section{Greek}

$\alpha$

elliptic blending parameter

$\delta$

boundary layer thickness

$\delta_{T}$

passive scalar boundary layer thickness

$\delta_{T z}$

$\Delta$

span-wise passive scalar boundary layer thickness

$\delta_{i j}$

$\varepsilon$

filter width

identity tensor

turbulence energy dissipation rate

$\theta$

passive scalar, temperature, concentration

$v$

kinematics viscosity

$\tau_{i j}$

stress tensor

$\phi_{i j}$

velocity-pressure gradient correlation

Acronyms

EB-RSM

Elliptic Blending Reynolds Stress Model

EB-GGDH

Elliptic Blending GGDH

GGDH

Generalized Gradient Diffusion Hypothesis 


$\begin{array}{ll}\text { LES } & \text { Large Eddy Simulation } \\ \text { RANS } & \text { Reynolds-Averaged Navier-Stokes } \\ \text { RSM } & \text { Reynolds Stress Model } \\ \text { SGDH } & \text { Simple Gradient Diffusion Hypothesis } \\ \text { sgs } & \text { subgrid scale }\end{array}$

\section{INTRODUCTION}

Heat transfer from discrete sources within a turbulent boundary layer is encountered in many and diverse engineering applications. Two-dimensional (2D) heat transfer is often studied for heated elements with finite stream-wise length and infinite lateral extent [1-5]. The effect of the lateral edges is rarely explored. In the context of electronic cooling, Baker [6] noticed that for small, discrete sources twodimensional correlations give heat fluxes as much as an order of magnitude smaller than those found experimentally, and speculated that the difference was due to conduction and edge effects. Only a few other studies [7-9] reported the effects of finite size on the local Nusselt number of discrete sources. Recently, Taliaferro et al. [10] investigated the heat transfer near a lateral edge of a flat plate in a laminar boundary layer flow. Using a conduction model, they were able to describe the local variation of heat transfer for fluids of a variety of Prandtl numbers, unheated starting lengths, and boundary conditions. Taliaferro et al. [11] extended the analysis to a finite width plate in a turbulent boundary layer, by transforming the heat transfer problem into an analogous mass transfer one using the naphthalene sublimation technique. Mass transfer experiments are ideally suited to studying fluxes from discrete sources due to the lack of losses to the substrate and ability to generate accurate local flux measurements near the lateral edge. A review of the naphthalene sublimation technique can be found in Goldstein and Cho [12].

The aim of the present study is to numerically reproduce the experimental results of Taliaferro et al. [11]. Studying such problems numerically requires an adequate modelling of the Reynolds stresses and the turbulent heat/mass flux near the wall. Wall-resolved large eddy simulations (LES) allow the accurate capture of the turbulent boundary layer and will be used here as a benchmark for ReynoldsAveraged Navier-Stokes (RANS) methods.

For a good modelling of the near-wall dynamics a so-called low-Reynolds RANS turbulence model is needed, i.e. a model that can represent the boundary layer turbulence down to the wall. Durbin's $\overline{v^{2}}$ $f$ model, for example, showed the right behaviour for the two-dimensional Stanton number of a turbulent boundary layer with an unheated starting length [13], when compared to Reynolds's experiments [14], with an eddy-diffusivity model for the turbulent heat flux (i.e. constant turbulent Prandtl number $\mathrm{Pr}_{\mathrm{t}}$ ). Two-equation models usually rely on the assumption of isotropic turbulence, which is not true in the boundary layer. In low-Reynolds number modelling, they are then constructed to capture only one component of the Reynolds stress tensor, which depends on a wall-normal turbulent 
viscosity. In thermal applications this component is mainly responsible for the wall-normal flux. This means that, if the stream-wise or span-wise contributions are not negligible, a model like this has significant limitations. A Reynolds Stress Model (RSM) is necessary to capture the contributions in the three directions, as will be discussed in section 2.2.

Similarly, in flows with strong dissimilarity between velocity and temperature fields the common assumption of a constant $\mathrm{Pr}_{\mathrm{t}}$ has limitations. Some numerical studies tried to improve the modelling by turning to two-equation models for heat transport [15-18]. However, the resulting turbulent diffusivity usually corresponds to the wall-normal component. A model for the complete three-dimensional turbulent heat flux vector is needed if the other two components are relevant [19]. A common choice is the anisotropic eddy diffusivity model known as Generalized Gradient Diffusion Hypothesis (GGDH) [20], which, however, does not take into account the wall effects.

A fully three-dimensional and low-Reynolds model for both the Reynolds stresses and the turbulent heat/mass flux is discussed in section 2.3. In the present work, LES and RANS simulations of the heat/mass transfer from a finite width plate in turbulent flow are compared to experimental results [11], with focus on the analysis of the turbulent terms that are responsible for the relative increase of the transfer coefficient at the lateral edge.

The paper is structured as follows. Section 2 defines the numerical methods, the LES and RANS models, and the computational details. Simulation results are discussed in Sections 3. Concluding remarks are provided in Section 4.

\section{NUMERICAL METHOD}

\subsection{LES governing equations}

The Large Eddy Simulation (LES) approach allows to solve the large-scale turbulent structure and to model the small-scale ones through a spatial filtering of the Navier-Stokes equations, leading to a reduction of the computational costs but capturing the fluid dynamics. If $a\left(t, x_{i}\right)$ is a generic field, function of time $t$ and spatial coordinate $x_{i}$, it is possible to define a grid-scale filtered field $\tilde{a}\left(t, x_{i}\right)$

$$
\tilde{a}\left(t, x_{i}\right)=\int_{\Omega} a\left(t, \xi_{i}\right) g\left(x_{i}-\xi_{i}, \Delta\right) d^{3} \xi_{i}
$$

The application of the filtering approach to the conservation equations, expressed in non-dimensional form, allows obtaining the following:

continuity equation

$$
\frac{\partial \tilde{u}_{i}}{\partial x_{i}}=0
$$

momentum equation 


$$
\frac{\partial \tilde{u}_{i}}{\partial t}+\frac{\partial}{\partial x_{j}}\left(\tilde{u}_{i} \tilde{u}_{j}+\tilde{p} \delta_{i j}-\frac{2}{\operatorname{Re}} \tilde{S}_{i j}\right)=\frac{\partial \tau_{i j}^{s s s}}{\partial x_{j}}
$$

passive scalar equation

$$
\frac{\partial \tilde{\theta}}{\partial t}+\frac{\partial}{\partial x_{k}}\left(\tilde{\theta} \cdot \tilde{u}_{k}-\frac{1}{\operatorname{Re}} \frac{1}{\operatorname{Pr}} \frac{\partial \tilde{\theta}}{\partial x_{k}}\right)=-\frac{\partial J_{k}^{s g s}}{\partial x_{k}}
$$

where $S_{i j}$, the rate of shear tensor, is defined as

$$
\tilde{S}_{i j}=\frac{1}{2}\left(\frac{\partial \tilde{u}_{i}}{\partial x_{j}}+\frac{\partial \tilde{u}_{j}}{\partial x_{i}}\right)-\frac{1}{3} \frac{\partial \tilde{u}_{k}}{\partial x_{k}} \delta_{i j}
$$

The sub-grid stress tensor, $\tau_{i j}^{\text {sgs }}$, is modeled with the "diffusive gradient” hypothesis

$$
\tau_{i j}^{s g s}=2 v_{s g s} \widetilde{S_{i j}}-\frac{2}{3} k_{s g s} \delta_{i j}
$$

Similarly, the sub-grid passive scalar flux vector, $J_{k}^{\text {sgs }}$, is

$$
J_{k}^{s g s}=-\frac{v_{s g s}}{\operatorname{Pr}_{s g s}} \frac{\partial \tilde{\theta}}{\partial x_{k}}
$$

where the sub-grid viscosity, $v_{s g s}$, is

$$
v_{s g s}=C_{s} \Delta^{2} \tilde{S}
$$

The sub-grid kinetic energy, $k_{s g s}$, is

$$
k_{\text {sgs }}=C_{I} \Delta^{2} \tilde{S}^{2}
$$

where the filtered shear rate, $\tilde{S}$, is

$$
\tilde{S}=\sqrt{2 \tilde{S}_{i j} \tilde{S}_{i j}}
$$

and the filter width, $\Delta$, is

$$
\Delta=\sqrt{\Delta_{k} \Delta_{k}}
$$

The dynamic Smagorinsky model, developed in [21] and successfully adopted in OpenFOAM in [2223], is employed.

\section{$2.2 \quad$ RANS modelling}

Within a turbulent boundary layer, the transport of heat and momentum from a surface is strongly influenced by the region immediately next to the surface. The presence of the wall suppresses the normal component of turbulent intensity within this region [24] (wall blockage), thus reducing heat and momentum transfer between wall and fluid. The resulting anisotropy is not accounted for by standard models, which are therefore not valid in the near-wall region and typically require the use of wall functions. An alternative method to wall functions was proposed by Durbin [25] in the $\overline{v^{\prime 2}}-f$ model, 
based on the solution of an Elliptic Relaxation equation for the velocity-pressure gradient correlation tensor involved in the Reynolds-stress transport equation. Its Reynolds stress model adaptation [26] was simplified by Manceau and Hanjalic [27], who proposed the Elliptic Blending approach, in which the six Elliptic Relaxation equations are replaced by a single equation for a wall-sensitive non-dimensional scalar $\alpha$.

Manceau [28] recently revisited various modifications of the original Elliptic Blending Reynolds Stress Model (EB-RSM) proposed by several authors [29-31], and formulated a reference EB-RSM, which was implemented in OpenFOAM for the present study. The model transport equations for the turbulent stress tensor and the energy dissipation rate, which constitute a second-moment closure, can be written in a general form:

$$
\begin{gathered}
\frac{\mathrm{Du}_{i}^{\prime} u_{j}^{\prime}}{\mathrm{D} t}=P_{i j}+G_{i j}+D_{i j}^{v}+D_{i j}^{T}+\phi_{i j}^{*}-\varepsilon_{i j} \\
\frac{\mathrm{D} \varepsilon}{\mathrm{D} t}=\frac{C_{\varepsilon 1}^{\prime} P_{k}-C_{\varepsilon 2} \varepsilon}{T}+\frac{\partial}{\partial x_{l}}\left[\frac{C_{\mu}}{\sigma_{\varepsilon}} \overline{u_{l}^{\prime} u_{m}^{\prime}} T \frac{\partial \varepsilon}{\partial x_{m}}\right]+v \frac{\partial^{2} \varepsilon}{\partial x_{k} \partial x_{k}}
\end{gathered}
$$

where $P_{i j}, D_{i j}^{v}, \phi_{i j}^{*}$ and $\varepsilon_{i j}$ stand for the production, the molecular diffusion, the velocity-pressure gradient correlation and the dissipation tensor, respectively. $D_{i j}^{T}=\frac{\partial}{\partial x_{l}}\left(\frac{C_{\mu}}{\sigma_{k}} \overline{u_{l}^{\prime} u_{m}^{\prime}} T \frac{\partial \overline{u_{i}^{\prime} u_{j}^{\prime}}}{\partial x_{m}}\right)$ is the turbulent diffusion term. $G_{i j}=-2 \omega_{k}\left(\epsilon_{i k m} \overline{u_{j}^{\prime} u_{m}^{\prime}}+\epsilon_{j k m} \overline{u_{i}^{\prime} u_{m}^{\prime}}\right)$ is the redistribution term arising from the Coriolis acceleration, where $\omega$ is the rotation axial vector.

The elliptic relaxation concept is adopted to blend the homogeneous (away-from-the-wall) and the near-wall models of $\phi_{i j}^{*}$

$$
\phi_{i j}^{*}=\left(1-\alpha^{3}\right) \phi_{i j}^{w}+\alpha^{3} \phi_{i j}^{h}
$$

where $\alpha$ is the elliptic blending parameter, obtained by solving the elliptic differential equation

$$
\alpha-L^{2} \nabla^{2} \alpha=1
$$

For the homogeneous part of the pressure scrambling term, $\phi_{i j}^{h}$, the model of Speziale et al. [32] is adopted

$$
\begin{aligned}
\phi_{i j}^{h} & =-\left(g_{1}+g_{1}^{*} \frac{P_{k}}{\varepsilon}\right) \varepsilon b_{i j}+\left(g_{3}-g_{3}^{*} \sqrt{b_{k l} b_{k l}}\right) k S_{i j}+ \\
& +g_{4} k\left(b_{i k} S_{j k}+b_{j k} S_{i k}-\frac{2}{3} b_{l m} S_{l m} \delta_{i j}\right)+ \\
& +g_{5} k\left(b_{i k} W_{j k}+b_{j k} W_{i k}\right)
\end{aligned}
$$

where

$$
\begin{gathered}
b_{i j}=\frac{\overline{u_{i}^{\prime} u_{j}^{\prime}}}{2 k}-\frac{1}{3} \delta_{i j} \\
S_{i j}=\frac{1}{2}\left(\frac{\partial U_{i}}{\partial x_{j}}+\frac{\partial U_{j}}{\partial x_{i}}\right)
\end{gathered}
$$




$$
W_{i j}=\frac{1}{2}\left(\frac{\partial U_{i}}{\partial x_{j}}-\frac{\partial U_{j}}{\partial x_{i}}\right)+\epsilon_{m j i} \omega_{m}
$$

For the near-wall part, $\phi_{i j}^{w}$, the model fully satisfies the exact wall limits and stress budget:

$$
\phi_{i j}^{w}=-5 \frac{\varepsilon}{k}\left[\overline{u_{i}^{\prime} u_{k}^{\prime}} n_{j} n_{k}+\overline{u_{j}^{\prime} u_{k}^{\prime}} n_{i} n_{k}-\frac{1}{2} \overline{u_{k}^{\prime} u_{l}^{\prime}} n_{k} n_{l}\left(n_{i} n_{j}+\delta_{i j}\right)\right]
$$

where the unit wall-normal vector is evaluated from

$$
\mathbf{n}=\frac{\nabla \alpha}{\|\nabla \alpha\|}
$$

The dissipation rate of the stress tensor is also expressed in terms of the homogeneous and near-wall variables, blended via the same elliptic function $\alpha$

$$
\varepsilon_{i j}=\left(1-\alpha^{3}\right) \frac{\overline{u_{i}^{\prime} u_{j}^{\prime}}}{k} \varepsilon+\frac{2}{3} \alpha^{3} \varepsilon \delta_{i j}
$$

For the viscous effect very close to the wall, Durbin's approach is adopted, which uses the conventional high-Re-number turbulence length and time scales away from a wall, but with imposed Kolmogorov scales as the lower bounds

$$
\begin{gathered}
L=C_{L} \max \left[\frac{k^{3 / 2}}{\varepsilon}, C_{\eta}\left(\frac{v^{3}}{\varepsilon}\right)^{1 / 4}\right] \\
T=\max \left[\frac{k}{\varepsilon}, C_{T}\left(\frac{v}{\varepsilon}\right)^{1 / 2}\right]
\end{gathered}
$$

Exact wall boundary conditions are used for all variables

$$
U_{i}=0 ; \quad \overline{u_{i}^{\prime} u_{j}^{\prime}}=0 ; \quad \varepsilon=2 v \lim _{y \rightarrow 0} \frac{k}{y^{2}} ; \quad \alpha=0
$$

The model coefficients are

$$
\begin{gathered}
g_{1}=3.4 ; \quad g_{1}^{*}=1.8 ; \quad g_{3}=0.8 ; \quad g_{3}^{*}=1.3 ; \quad g_{4}=1.25 \\
g_{5}=0.4 ; \quad C_{\mu}=0.21 ; \quad \sigma_{k}=1.0 ; \quad C_{T}=6.0 ; \\
C_{L}=0.133 ; \quad C_{\eta}=80.0 ; \quad C_{\varepsilon 1}=1.44 ; \quad C_{\varepsilon 2}=1.9 ; \\
A_{1}=0.065 ; \quad C_{\varepsilon 1}^{\prime}=C_{\varepsilon 1}\left[1+A_{1}\left(1-\alpha^{3}\right) \frac{P_{k}}{\varepsilon}\right] ; \quad \sigma_{\varepsilon}=1.15
\end{gathered}
$$

\subsection{Turbulent heat/mass transfer modelling}

The closure of the energy equation

$$
\frac{\mathrm{D} \bar{\theta}}{\mathrm{D} t}=\frac{\partial}{\partial x_{j}}\left(\frac{v}{\operatorname{Pr}} \frac{\partial \bar{\theta}}{\partial x_{j}}-\overline{u_{j}^{\prime} \theta^{\prime}}\right)
$$

can take advantage of the elliptic blending of the Reynolds stresses for a more accurate modelling of the turbulent heat flux $\overline{u_{j}^{\prime} \theta^{\prime}}$. Shin et al. [34] defined a transport equation for $\overline{u_{j}^{\prime} \theta^{\prime}}$, based on the elliptic relaxation equation, leading to the Elliptic Blending Differential Flux Model (EB-DFM). However, such an approach might not be optimal due to its high level of complexity. A lower modelling level for the 
turbulent heat flux model is usually considered acceptable, especially if the thermal field is passive. Dehoux et al. [35] thus derived from the EB-DFM a simpler low-Reynolds algebraic flux model, known as Elliptic Blending Generalized Gradient Diffusion Hypothesis (EB-GGDH):

$$
\overline{u_{j}^{\prime} \theta^{\prime}}=-C_{\theta}\left[\frac{k}{\varepsilon} \overline{u_{i}^{\prime} u_{j}^{\prime}} \frac{\partial \bar{\theta}}{\partial x_{j}}+\gamma \overline{u_{k}^{\prime} \theta^{\prime}} n_{i} n_{j}\right]
$$

where

$$
\begin{gathered}
C_{\theta}=\frac{0.68}{3 \alpha^{3}+\left(1-\alpha^{3}\right) C_{\varepsilon}} \\
\gamma=\left(1-\alpha^{3}\right)\left[1+C_{\varepsilon}\right] \\
C_{\varepsilon}=\frac{1}{2}\left(1+\frac{1}{\operatorname{Pr}}\right)
\end{gathered}
$$

This model only differs from the standard GGDH by the additional term $\overline{\overline{\gamma u_{k}^{\prime} \theta^{\prime} n_{i} n_{j}}}$, which sensitizes the model to the orientation of the wall, and by the fact that the coefficient $C_{\theta}$ is now dependent on a blending function $\alpha_{\theta}$, which, in the present study, has been assumed equal to $\alpha$ of the EB-RSM, following Shin et al. [34].

Without taking into account the wall effect, the EB-GGDH model reduces to the well-known form of the GGDH:

$$
\overline{u_{j}^{\prime} \theta^{\prime}}=-C_{\theta} \frac{k}{\varepsilon} \overline{u_{i}^{\prime} u_{j}^{\prime}} \frac{\partial \bar{\theta}}{\partial x_{j}}
$$

where $C_{\theta}=0.235[35]$.

The standard isotropic eddy-diffusivity model, also known as the Simple Gradient Diffusion Hypothesis (SGDH), can be obtained by assuming that $i=j$ (i.e., heat flux is function of the temperature gradient in that direction):

$$
\overline{u_{j}^{\prime} \theta^{\prime}}=-\frac{v_{t}}{\operatorname{Pr}_{t}} \frac{\partial \bar{\theta}}{\partial x_{j}}
$$

where the turbulent Prandtl number can be obtained by previously defined constants

$$
\operatorname{Pr}_{t}=\frac{C_{\mu}}{C_{\theta}} \approx 0.9
$$

In the following sections we will compare the results obtained with these three heat flux models, SGDH, GGDH and EB-GGDH (all implemented in OpenFOAM for the present study), as well as with Large Eddy Simulations.

\section{$2.4 \quad$ Computational details}

The LES simulations are carried out with the finite-volume solver, pisoFoamPS, developed in [36-37] and implemented in the open-source code OpenFOAM, to solve the Navier-Stokes equations with a passive scalar equation. pisoFoamPS is a transient solver for incompressible turbulent flow, which uses the PISO algorithm. The RANS simulations were performed in steady state with an analogous 
simpleFoamPS solver, which uses the SIMPLE algorithm and solves an additional passive scalar equation.

In the following sections we will mostly refer to the passive scalar field as the temperature field, but the concentration field of the equivalent mass transfer problem would behave the same way, as will be shown by comparison with the experimental results [11], obtained with the naphthalene sublimation technique.

All the variables are presented in a non-dimensional form. The reference values are the thickness $\delta$ of the velocity boundary layer at the beginning of the heated plate, the free-stream velocity and temperature. Following Abe et al. [38], all the variables expressed with the ${ }^{+}$sign are normalized by the friction velocity $U_{\tau}$ and the friction temperature $T_{\tau}=Q_{w} / \rho c_{p} U_{\tau}$, where $Q_{w}$ is the local surface heat flux.

Figure 1 shows a sketch of the simulation domain. The 3D grid was generated with blockMesh, the OpenFOAM utility for mesh generation. The geometry consists of a rectangular box with $-10<x<22$, $0<y<3$ and $-1.5<z<1.5$. The heated plate is the part of the wall limited by $x>0$ and $z>0$. The grid is made of $256 \times 64 \times 128$ cells. The resulting spatial resolution is: $y^{+} \approx 0.2$ (wall-adjacent cell center), $\Delta x^{+} \approx 40$, and $\Delta z^{+} \approx 8$. The choice of a $y^{+}$well below 1 was driven by the more stringent requirements of the thermal boundary layer.

Fig. 1 - Case domain showing orientation and boundary layer development

Two experimental values of the free-stream velocity [11] are used, corresponding to a Reynolds number $R e_{x^{\prime}}$ on the leading edge of the heated plate of about $1.56 \times 10^{5}$ (Case 1 ) and $4.81 \times 10^{5}$ (Case 2), where $x^{\prime}$ is the distance from the virtual origin of the turbulent boundary layer.

The boundary conditions for the RANS simulations are as follows. A no-slip wall condition is set at the bottom boundary, and a slip condition at the top and side boundaries. A pressure outlet condition is imposed at the outflow. Inlet conditions for all variables were extracted from a $2 \mathrm{D}$ calculation of a developing boundary layer.

The boundary conditions for the LES simulations are as follows. A no-slip wall condition is set at the bottom boundary, and a slip condition at the top one. The side boundaries are treated as periodic. A pressure outlet condition is imposed at the outflow. Inflow velocity data are generated following Lund's parallel-flow recycling method [39-40]: a simulation is performed simultaneously with the main case, on a truncated version of the case grid, extending one boundary layer thickness $\delta$ in the wall-normal direction and $5 \delta$ in the stream-wise direction, with periodic boundary conditions in the stream-wise and span-wise directions and a slip boundary condition at the upper boundary. This approach is economical and has the advantage that the inflow turbulence is fully developed. A development section of $10 \delta$ is considered sufficient to establish the correct boundary layer spatial growth characteristics [41]. 
For both RANS and LES calculations, the boundary conditions for the temperature are $\theta=1$ at the inlet, $\theta=0$ on the heated plate and adiabatic on the unheated wall.

\section{RESULTS}

The problem studied in the present work is visualized in Figure 2, where instantaneous LES results are shown. The finite width heated plate ( $\theta=0)$ is here depicted with the heat transfer/mass transfer analogy as a white plate "releasing" mass to the flow, similarly to the experiments conducted with the naphthalene sublimation technique [11]. More specifically, the white iso-surface is defined for $\theta=0.1$, qualitatively showing the mixing of the temperature/concentration field within the turbulent boundary layer. The rest of the solid surface, shown in black, is characterized by an adiabatic boundary condition. A vertical cut in the $x-y$ plane presents the velocity magnitude contours in rainbow colour-map, revealing the developing turbulent boundary layer.

Fig. 2 - Iso-surface of $\theta=0.1$ in white, with the black adiabatic wall and a cut-plane showing boundary layer velocity contour in rainbow, from LES results of Case 2.

First- and second-order statistics of the boundary layer are of crucial importance for the correct numerical calculation of the heat/mass transfer. LES can be negatively affected by inadequate turbulence recycling techniques, while RANS can yield inaccurate results if the turbulence model is not appropriate. The following analysis aims at validating the LES (and the recycling method) with the experimental data, and at evaluating the presented RSM and turbulent heat flux models in comparison with the LES results.

Profiles of the mean velocity, obtained by EB-RSM and LES, are compared with the experimental measurements [11] in Figure 3. The data are taken at a section just downstream of the leading edge of the heated plate: $R e_{x^{\prime}}=1.65 \times 10^{5}$ for Case 1 and $R e_{x^{\prime}}=4.9 \times 10^{5}$ for Case 2 . The numerical predictions are in very good agreement. The EB-RSM model agrees well with the measurements, confirming the validity of Durbin's elliptic relaxation approach [24]. Thanks to the dynamic Smagorinsky model, The LES shows a very good agreement in Case 1, while it slightly overestimates the velocity in the logarithmic layer of Case 2 (i.e. at higher Reynolds number). This is likely to be due to a slightly insufficient development length of the turbulence recycling method. However, this discrepancy does not have a noticeable impact on the overall behavior of the flow. Moreover, as will be shown in the following analysis, the heat/mass transfer lateral effects are driven predominantly by the inner regions $\left(y^{+}<20\right)$ of the boundary layer, where agreement is very good in both cases.

Fig. 3 - Mean velocity profile. (a) Case 1; (b) Case 2. 
Figure 4 shows a comparison of the Reynolds stresses obtained from LES and RANS. The two methods are in very good agreement, especially for the shear stress $\overline{u^{\prime} v^{\prime}}$ and the vertical normal stress $\overline{v^{\prime} v^{\prime}}$, while $\overline{w^{\prime} w^{\prime}}$ presents a slight overprediction of RANS compared to LES. The difference in the peak value of the streamwise normal stress $\overline{u^{\prime} u^{\prime}}$ is thought to be due to the formulation of the elliptic blending parameter $\alpha$ and its dependence on the Reynolds number [28]. It is important to notice that, while LES is generally expected to capture the anisotropy of the turbulent boundary layer, most RANS models are not built to. The EB-RSM is not only able to calculate the different components of the Reynolds stress tensor, but the elliptic blending strategy makes it integrable down to the wall with only one additional equation.

Fig. 4 - Reynolds stress profiles from LES (symbols) and EB-RSM (lines). (a) Case 1; (b) Case 2.

Figure 5 shows the wall-normal temperature profiles, plotted on the centerline of the heated plate (i.e. far from the lateral edge), at $R e_{x^{\prime}}=1.8 \times 10^{5}$ for Case 1 and $R e_{x^{\prime}}=5.7 \times 10^{5}$ for Case 2 . Three RANS simulations with different turbulent heat flux models (SGDH, GGDH and EB-GGDH) are compared to LES. It is apparent that the presence of an unheated starting length produces a thermal boundary layer profile that is quite different from the universal one. Therefore, the assumption of isotropic eddydiffusivity of SGDH (i.e. constant turbulent Prandtl number Prt) is not expected to hold. Even though the GGDH introduces anisotropy, as discussed in Section 2.3, in this location the wall-normal heat flux is dominant, and the temperature profile consequently coincides with that calculated with the SGDH. Accounting for the near-wall effects (EB-GGDH) slightly modifies the prediction of the wall-normal flux (and therefore the friction temperature), but the LES modeling seems to have the opposite effect. The reason for this discrepancy lies in the difficulty of modelling velocity and temperature fields that are so dissimilar, due to the strong effect of even small differences in the wall-normal turbulent heat flux $\overline{v^{\prime} \theta^{\prime}}$, as can be seen in Fig. 6. Here the different models yield very similar results in the viscous sublayer and in the logarithmic region, while they present a slight difference in the buffer region $\left(y^{+} \approx\right.$ 10), which must therefore be responsible for the different prediction of Fig. 5.

Fig. 5 - Mean temperature profile. (a) Case 1; (b) Case 2.

Fig. 6 - Wall-normal turbulent heat flux. (a) Case 1; (b) Case 2.

The effects of this discrepancy are noticeable in the heat transfer coefficient along the plate in the stream-wise direction, expressed in Figure 7 in the form of Stanton number, defined as

$$
\mathrm{St}=\frac{\mathrm{Nu}}{\mathrm{Re} \cdot \operatorname{Pr}}=\frac{\mathrm{Sh}}{\mathrm{Re} \cdot \mathrm{Sc}}
$$


The former formulation is its definition for the heat transfer problem, whereas the latter is for the analogous mass transfer. Nu and Pr are the Nusselt and Prandlt numbers, corresponding through the heat/mass transfer analogy to the Sherwood number, Sh, and the Schmidt number, Sc, respectively. The comparison of the centerline Stanton number, $\mathrm{St}_{c}$, with the experimental data [11] shows a good overall prediction with all turbulent heat transfer models. The EB-GGDH seems to be in better agreement for Case 1, while LES better matches the experiments in Case 2. This is mainly the result of the abovementioned discrepancy of Figure 6, revealing how sensitive this problem is to minor changes in the turbulence modelling.

Fig. 7 - Stanton number on the centerline of the plate. (a) Case 1; (b) Case 2.

As soon as the span-wise effects of the lateral edge are no longer negligible, the differences in modeling become more apparent. Figure 8 shows the span-wise turbulent heat flux component, $\overline{w^{\prime} \theta^{\prime}}$, predicted by the simulations, plotted at $z=0$. It is now clear that the SGDH uses the same approach as for the wall-normal direction, resulting in an unwanted wall blockage: the span-wise heat flux becomes negligible in the viscous sublayer. On the other hand, the GGDH introduces anisotropy and thus captures a stronger behavior in the viscous and buffer layers. Finally, the EB-GGDH uses a near-wall modeling which further increases the span-wise heat flux in these regions. Its agreement with the LES results, especially in Case 2, confirms the validity of this model in problems where the 3D near-wall effects are strong.

Fig. 8 - Span-wise turbulent heat flux. (a) Case 1; (b) Case 2.

Experimental validation of these lateral effects can be found in Fig. 9. Here the relative increase along $z$ of the numerical Stanton number at the lateral edge of the plate is compared to the experimental results of the mass transfer from the naphthalene plate, at positions $R e_{x^{\prime}}=1.8 \times 10^{5}$ for Case 1 and $R e_{x^{\prime}}=5.7 \times 10^{5}$ for Case 2 . The SGDH confirms the underestimation of the span-wise heat $/$ mass transfer. The GGDH shows an increased lateral effect, thanks to its anisotropic eddy diffusivity. The LES and the EB-GGDH are the best option to capture this lateral increase. The EB-GGDH is even in better agreement with experiments than LES in Case 1, where the span-wise turbulent heat flux of LES (see Fig. 8) is less pronounced.

Fig. 9 - Relative increase of the Stanton number at the lateral edge. (a) Case 1; (b) Case 2.

Fig. 10 - Span-wise temperature boundary layer thickness on the unheated plate (a) Case 1; (b) Case 2. 
The effects of the span-wise heat transfer are also revealed from the numerical results on the adjacent adiabatic wall. A $90 \%$ temperature boundary layer thickness, $\delta_{T z}$, can be defined in the span-wise direction from the lateral edge of the plate, as depicted in Fig. 1. In Figure 10, as expected, the SGDH and GGDH models underestimate the lateral extent of the temperature boundary layer, while the EBGGDH and LES show a $\delta_{\text {Tz }}$ of the order of 0.1 (10\% of the velocity boundary layer thickness $\delta$ ). Their very good agreement, especially in Case 2, is a direct consequence of the accurate modelling of the span-wise turbulent heat flux component, $\overline{w^{\prime} \theta^{\prime}}$, presented in Figure 8.

\section{CONCLUSION}

Heat transfer from discrete sources is strongly affected by lateral effects. The presence of an unheated region in the immediate vicinity of the side edge produces an increased local heat transfer coefficient, which is directly linked to the near-wall anisotropy of the flow. In particular, in turbulent flow the spanwise component of the turbulent heat flux is responsible for most of the lateral effects. A correct modelling of this component is therefore crucial for an accurate numerical prediction of the local heat transfer. RANS models often rely on several assumptions that are inadequate for this purpose. Isotropic eddy-diffusivity models (SGDH) are not able to capture the anisotropy of the turbulent heat flux. A more advanced approach, based on the Generalized Gradient Diffusion Hypothesis (GGDH), introduces anisotropy by using the full Reynolds stress tensor, therefore requiring a second-moment closure (Reynolds Stress Model), instead of the more common two-equation turbulence model. However, even in combination with low-Reynolds RSM models (such as the EB-RSM), the GGDH still assumes a strong similarity between velocity and temperature fields. When this assumption does not hold, nearwall contributions become more important. Near-wall sensitivity of the turbulent heat flux can be improved with an elliptic relaxation equation, such as the one used in the EB-RSM, leading to the EBGGDH model.

This study presents numerical predictions obtained with all the abovementioned models. Large Eddy Simulations are used as a benchmark. By means of the heat/mass transfer analogy, results are compared to mass transfer experiments conducted with the naphthalene sublimation technique. The wall-normal component of the turbulent heat flux, far from the lateral edge, is shown to be correctly captured by all numerical approaches, which confirms the suitability of basic models in simple 2D configurations. On the contrary, the span-wise effects at the lateral edge are underestimated by the SGDH and GGDH, and only the EB-GGDH seems to yield results that are comparable to LES and to the experiments. These results indicate that in applications with strong three-dimensional effects and dissimilarity between velocity and temperature fields, the near wall anisotropy can be successfully modelled only by eddy resolving methods or by advanced turbulent heat flux models such as the EB-GGDH. 


\section{REFERENCES}

[1] S. Ramadhyani, D. F. Moffatt, and F. P. Incropera. Conjugate Heat Transfer from Small Isothermal Heat Sources Embedded in a Large Substrate. International Journal of Heat and Mass Transfer 28 (10) (1985) 1945-52.

[2] F. P. Incropera, J. S. Kerby, D. F. Moffatt, and S. Ramadhyani. Convection Heat Transfer from Discrete Heat Sources in a Rectangular Channel. International Journal of Heat and Mass Transfer 29 (7) (1986) 1051-58.

[3] K. D. Cole. Conjugate Heat Transfer from a Small Heated Strip. International Journal of Heat and Mass Transfer 40 (11) (1997) 2709-19.

[4] A. K. da Silva, S. Lorente, and A. Bejan. Optimal Distribution of Discrete Heat Sources on a Plate with Laminar Forced Convection. International Journal of Heat and Mass Transfer 47, (10-11) (2004) 2139-48.

[5] Gh. Juncu. Unsteady Conjugate Forced Convection Heat/Mass Transfer from a Finite Flat Plate. International Journal of Thermal Sciences 47 (8) (2008) 972-84.

[6] E. Baker. Liquid Cooling of Microelectronic Devices by Free and Forced Convection. Microelectronics Reliability 11 (2) (1972) 213-22.

[7] A. Ortega, and S. Ramanathan. On the Use of Point Source Solutions for Forced Air Cooling of Electronic Components - Part II: Conjugate Forced Convection From a Discrete Rectangular Source on a Thin Conducting Plate. Journal of Electronic Packaging 125 (2) (2003) 235.

[8] O. Wang, and Y. Jaluria. Three-Dimensional Conjugate Heat Transfer in a Horizontal Channel with Discrete Heating. Journal of Heat Transfer 126 (4) (2004) 642.

[9] P. S. Jakkareddy, and C. Balaji. A Non-Intrusive Technique to Determine the Spatially Varying Heat Transfer Coefficients in a Flat Plate with Flush Mounted Heat Sources. International Journal of Thermal Sciences 131 (2018) 144-59.

[10] M. E. Taliaferro, M. Angelino, F. Gori, and R. J. Goldstein. Local Heat Transfer on a Finite Width Surface with Laminar Boundary Layer Flow. Applied Thermal Engineering 101 (2016) 686-92.

[11] M. E. Taliaferro, F. Fassio, F. Gori, T. W. Simon, and R. J. Goldstein. Lateral Edge Effects on the Sherwood Number in Turbulent Flow Over a Flat Plate. Journal of Heat Transfer 140 (2) (2017) 023001.

[12] R. J. Goldstein, and H. H. Cho. A Review of Mass Transfer Measurements Using Naphthalene Sublimation. Experimental Thermal and Fluid Science 10 (4) (1995) 416-34.

[13] P. A. Durbin. Application of a Near-Wall Turbulence Model to Boundary Layers and Heat Transfer. International Journal of Heat and Fluid Flow 14 (4) (1993) 316-23.

[14] W. C. Reynolds, W. M. Kays, and S. J. Kline. Heat Transfer in the Turbulent Incompressible Boundary Layer, III - Arbitrary Wall Temperature and Heat Flux. NASA Memorandum (1958).

[15] T. P. Sommer, R. M. C. So, and Y. G. Lai. A Near-Wall Two-Equation Model for Turbulent Heat Fluxes. International Journal of Heat and Mass Transfer 35 (12) (1992) 3375-87. 
[16] M. S. Youssef, Y. Nagano, and M. Tagawa. A Two-Equation Heat Transfer Model for Predicting Turbulent Thermal Fields under Arbitrary Wall Thermal Conditions. International Journal of Heat and Mass Transfer 35 (11) (1992) 3095-3104.

[17] K. Abe, T. Kondoh, and Y. Nagano. A New Turbulence Model for Predicting Fluid Flow and Heat Transfer in Separating and Reattaching Flows - II. Thermal Field Calculations. International Journal of Heat and Mass Transfer 38 (8) (1995) 1467-81.

[18] H. Hattori, and Y. Nagano. Rigorous Formulation of Two-Equation Heat Transfer Model of Turbulence Using Direct Simulations. Numerical Heat Transfer, Part B: Fundamentals 33 (2) (1998) 153-80.

[19] K. Matsubara, A. Sakurai, T. Miura, and T. Kawabata. Spanwise Heat Transport in Turbulent Channel Flow With Prandtl Numbers Ranging From 0.025 to 5.0. Journal of Heat Transfer 134 (4) (2012) 041701.

[20] B. J. Daly, and F. H. Harlow. Transport Equations in Turbulence. Physics of Fluids 13 (11) (1970) 2634.

[21] M. Germano, U. Piomelli, P. Moin, and W. H. Cabot. A Dynamic Subgrid-Scale Eddy Viscosity Model. Physics of Fluids A: Fluid Dynamics 3 (7) (1991) 1760-1765.

[22] A. Boghi, M. Angelino, and F. Gori. Numerical Evidence of an Undisturbed Region of Flow in a Turbulent Rectangular Submerged Free Jet. Numerical Heat Transfer, Part A: Applications 70 (1) (2016) 14-29.

[23] M. Angelino, A. Boghi, and F. Gori. Numerical Solution of Three-Dimensional Rectangular Submerged Jets with the Evidence of the Undisturbed Region of Flow. Numerical Heat Transfer, Part A: Applications 70 (8) (2016) 815-30.

[24] J. C. R. Hunt, and J. M. R. Graham. Free-Stream Turbulence near Plane Boundaries. Journal of Fluid Mechanics 84 (2) (1978) 209.

[25] P. A. Durbin. Near-Wall Turbulence Closure Modeling without “Damping Functions”. Theoretical and Computational Fluid Dynamics 3 (1) (1991) 1-13.

[26] P. A. Durbin. A Reynolds Stress Model for Near-Wall Turbulence. Journal of Fluid Mechanics 249 (1) (1993) 465.

[27] R. Manceau, and K. Hanjalić. Elliptic Blending Model: A New near-Wall Reynolds-Stress Turbulence Closure. Physics of Fluids 14 (2) (2002) 744.

[28] R. Manceau. Recent Progress in the Development of the Elliptic Blending Reynolds-Stress Model. International Journal of Heat and Fluid Flow 51 (2015) 195-220.

[29] R. Manceau. An Improved Version of the Elliptic Blending Model Application to Non-Rotating and Rotating Channel Flows. In 4th International Symposium on Turbulence and Shear Flow Phenomena, 1 (2005) 259-64.

[30] L. Thielen, K. Hanjalić, H. Jonker, and R. Manceau. Predictions of Flow and Heat Transfer in Multiple Impinging Jets with an Elliptic-Blending Second-Moment Closure. International Journal of Heat and Mass Transfer 48 (8) (2005) 1583-98. 
[31] Y. Lecocq, R. Manceau, S. Bournaud, and L.-E. Brizzi. Modelling of the Turbulent Heat Fluxes in Natural, Forced and Mixed Convection Regimes. In Proc. 7th ERCOFTAC Int. Symp. on Eng. Turb. Modelling and Measurements (2008).

[32] C. G. Speziale, S. Sarkar, and T. B. Gatski. Modelling the Pressure-Strain Correlation of Turbulence: An Invariant Dynamical Systems Approach. Journal of Fluid Mechanics 227 (1) (1991) 245.

[33] F. Billard, and D. Laurence. A Robust $\mathrm{K}-\varepsilon^{-}-\mathrm{v} 2 / \mathrm{k}$ Elliptic Blending Turbulence Model Applied to near-Wall, Separated and Buoyant Flows. International Journal of Heat and Fluid Flow 33 (1) (2012) 45-58.

[34] J. K. Shin, J. S. An, Y. D. Choi, Y. C. Kim, and M. S. Kim. Elliptic Relaxation Second Moment Closure for the Turbulent Heat Fluxes. Journal of Turbulence 9 (3) (2008) 1-29.

[35] F. Dehoux, Y. Lecocq, S. Benhamadouche, R. Manceau, and L.-E. Brizzi. Algebraic Modeling of the Turbulent Heat Fluxes Using the Elliptic Blending Approach-Application to Forced and Mixed Convection Regimes. Flow, Turbulence and Combustion 88 (1-2) (2012) 77-100.

[36] I. Di Venuta, I. Petracci, M. Angelino, A. Boghi, and F. Gori. Numerical Simulation of Mass Transfer and Fluid Flow Evolution of a Rectangular Free Jet of Air. International Journal of Heat and Mass Transfer 117 (2018) 235-51.

[37] I. Di Venuta, A. Boghi, M. Angelino, and F. Gori. Passive Scalar Diffusion in Three-Dimensional Turbulent Rectangular Free Jets with Numerical Evaluation of Turbulent Prandtl/Schmidt Number. International Communications in Heat and Mass Transfer 95 (2018) 106-15.

[38] H. Abe, H. Kawamura, and Y. Matsuo. Surface Heat-Flux Fluctuations in a Turbulent Channel Flow up to $\operatorname{Re} \tau=1020$ with $\operatorname{Pr}=0.025$ and 0.71. International Journal of Heat and Fluid Flow 25 (3) (2004) 404-19.

[39] T. S. Lund. Large Eddy Simulation of a Boundary Layer with Concave Streamwise Curvature. In Annual Research Briefs, 91-99. Center for Turbulence Research (1993).

[40] T. S. Lund, and P. Moin. Large-Eddy Simulation of a Concave Wall Boundary Layer. International Journal of Heat and Fluid Flow 17 (3) (1996) 290-95.

[41] T. S. Lund, X. Wu, and K. D. Squires. Generation of Turbulent Inflow Data for SpatiallyDeveloping Boundary Layer Simulations. Journal of Computational Physics 140 (2) (1998) 23358. 


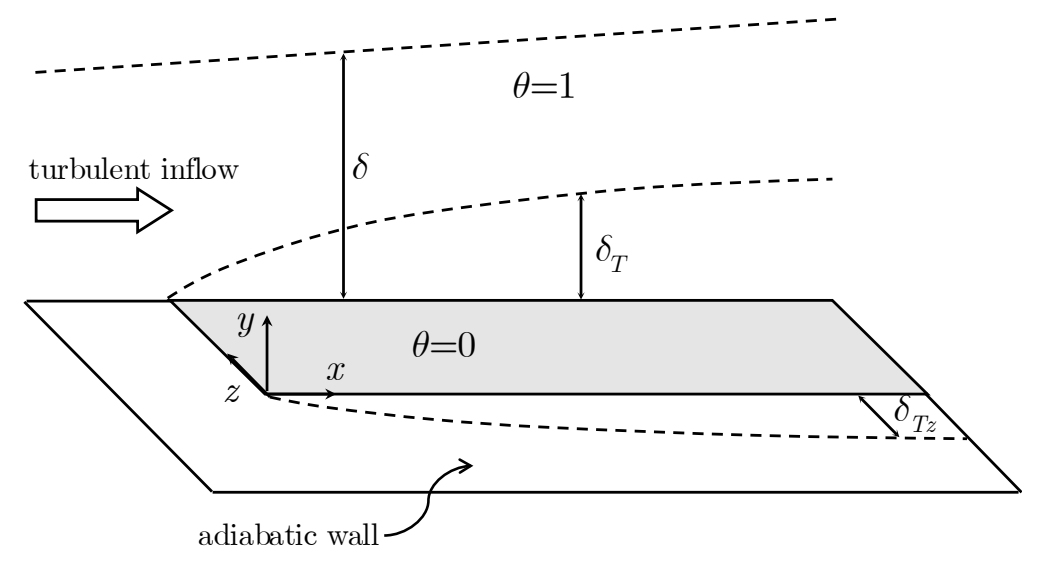

Fig. 1 - Case domain showing orientation and boundary layer development

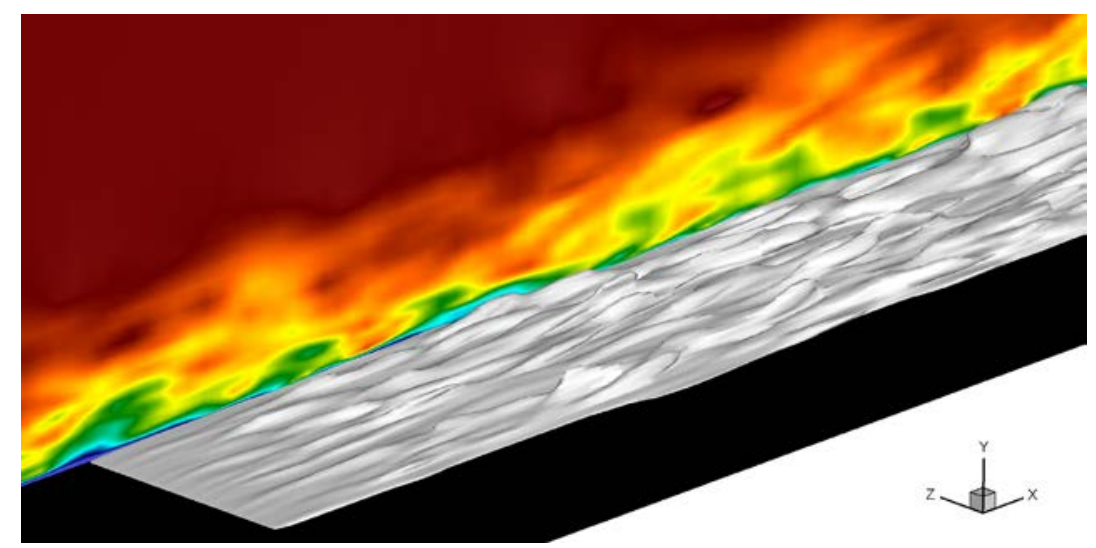

Fig. 2 - Iso-surface of $\theta=0.1$ in white, with the black adiabatic wall and a cut-plane showing boundary layer velocity contour in rainbow, from LES results of Case 2. 

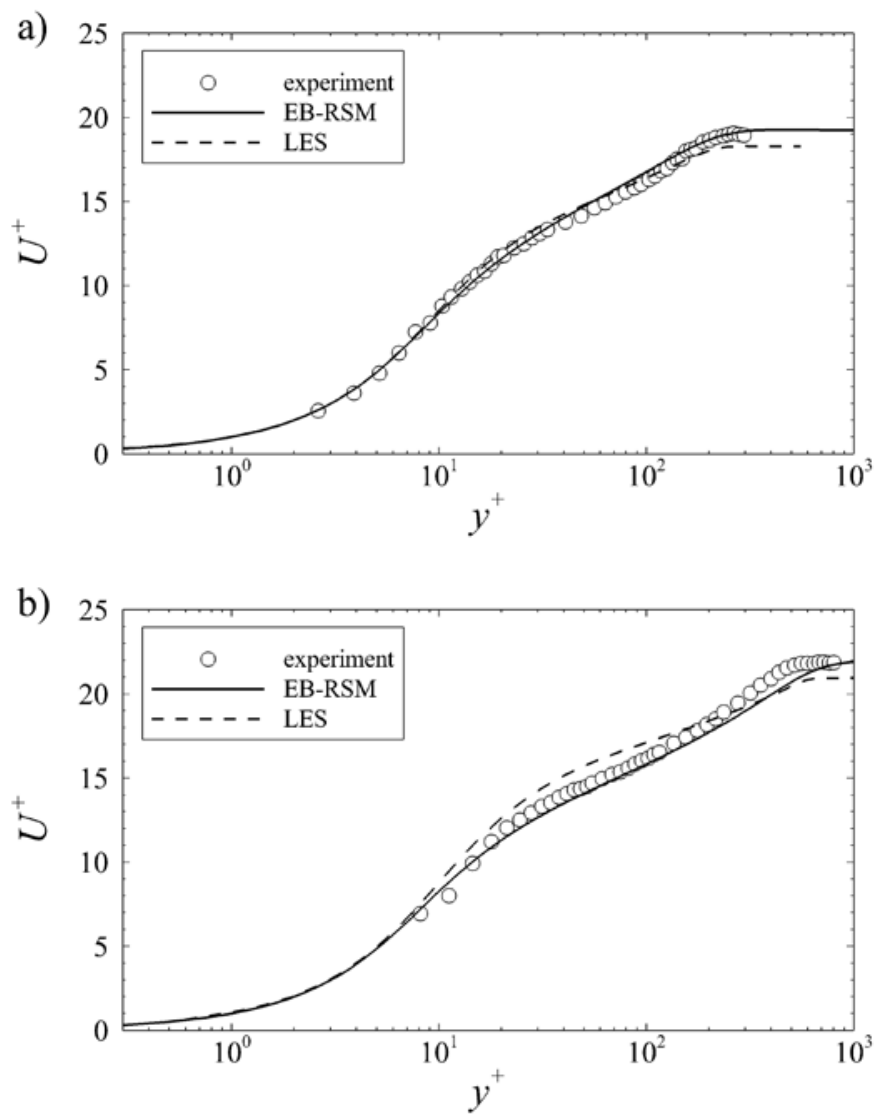

Fig. 3 - Mean velocity profile. (a) Case 1; (b) Case 2.
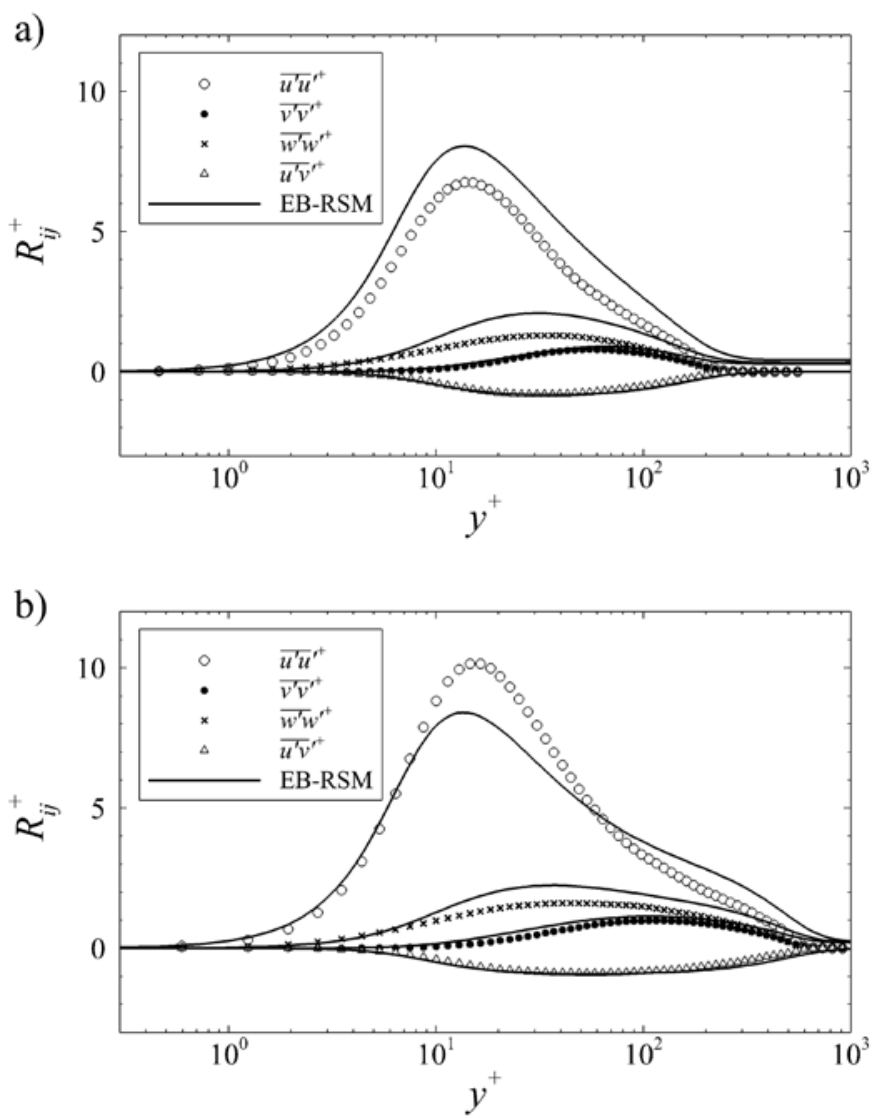

Fig. 4 - Reynolds stress profiles from LES (symbols) and EB-RSM (lines). (a) Case 1; (b) Case 2. 

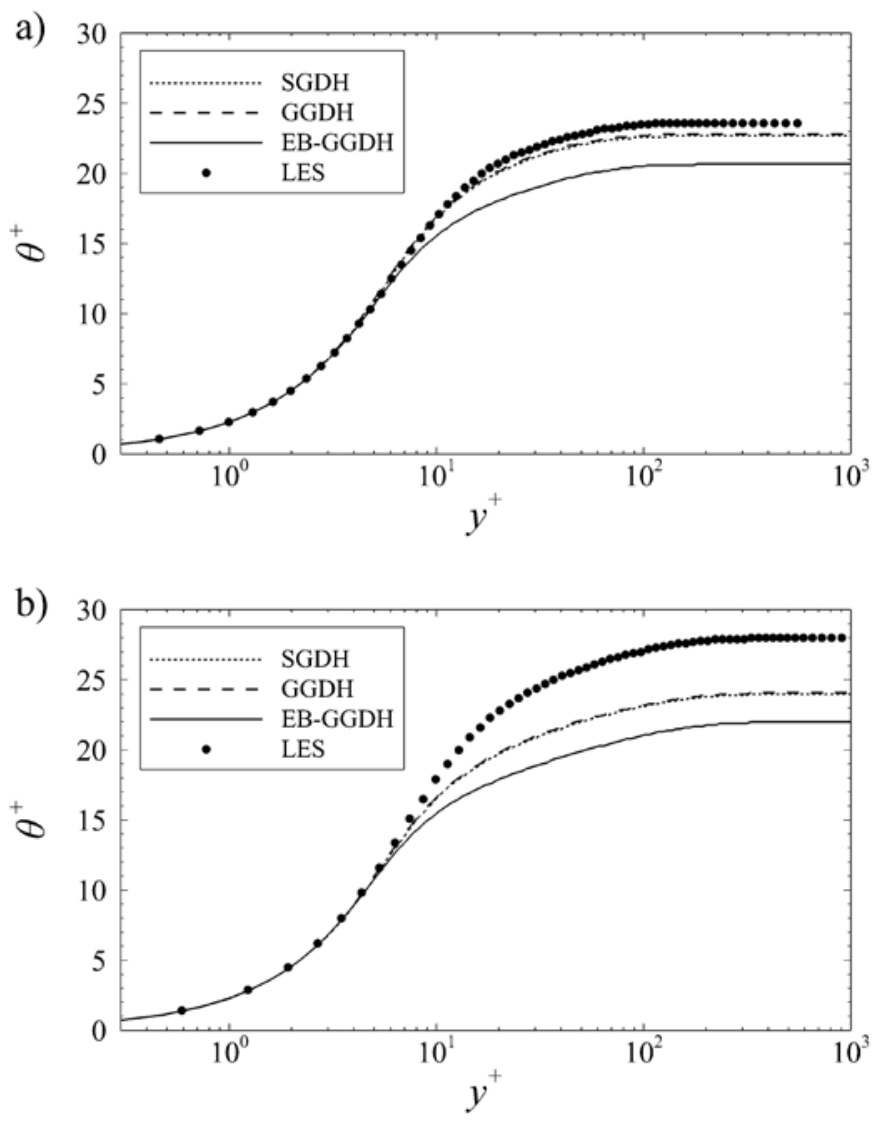

Fig. 5 - Mean temperature profile. (a) Case 1; (b) Case 2.
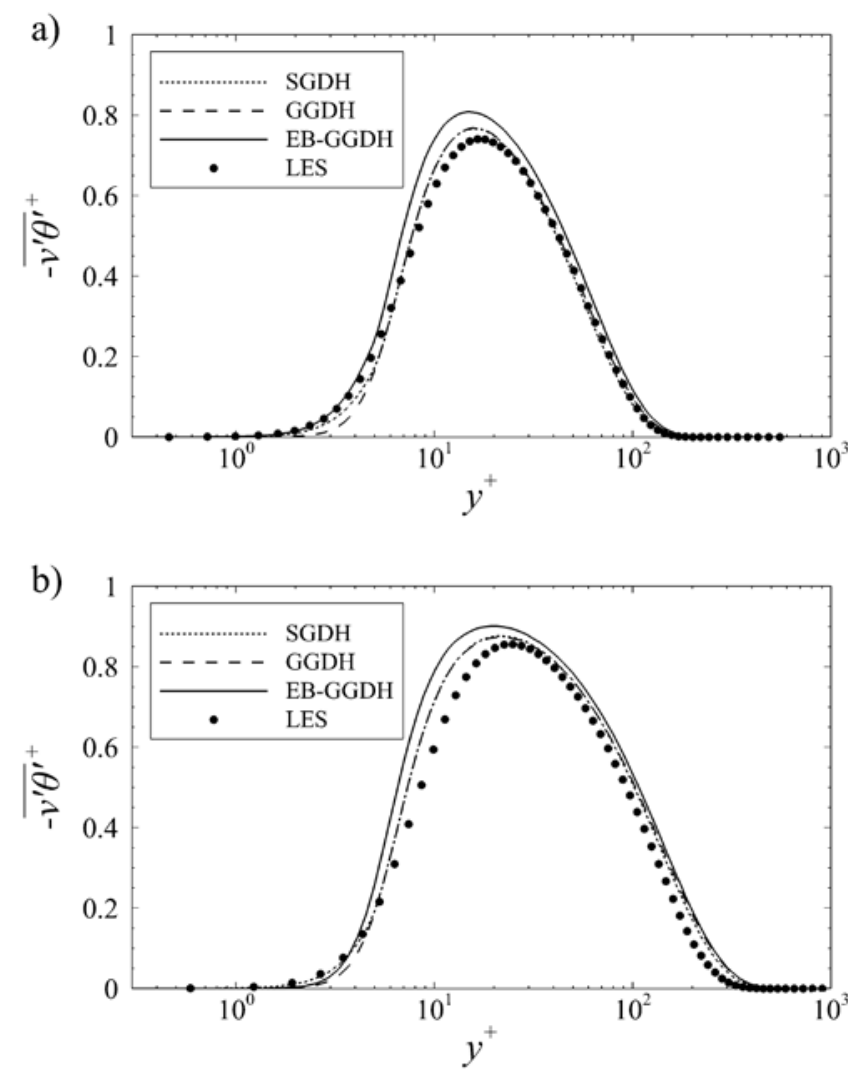

Fig. 6 - Wall-normal turbulent heat flux. (a) Case 1; (b) Case 2. 

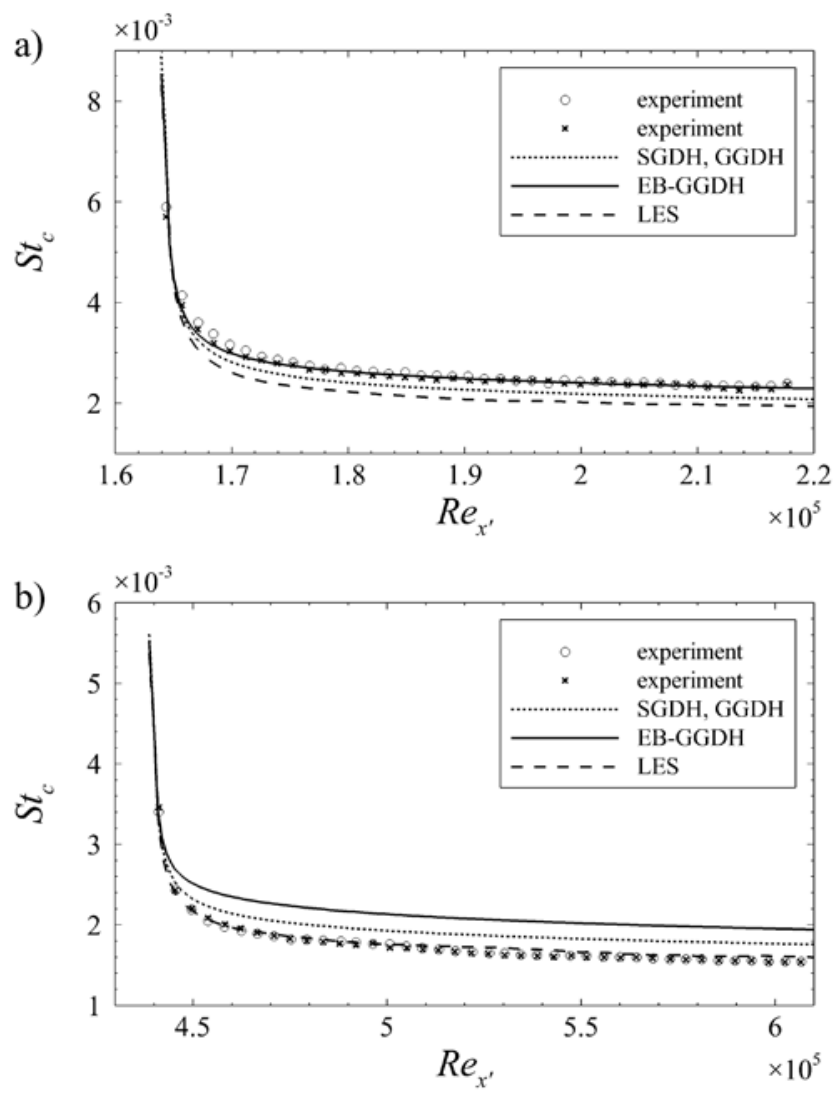

Fig. 7 - Stanton number on the centerline of the plate. (a) Case 1; (b) Case 2.
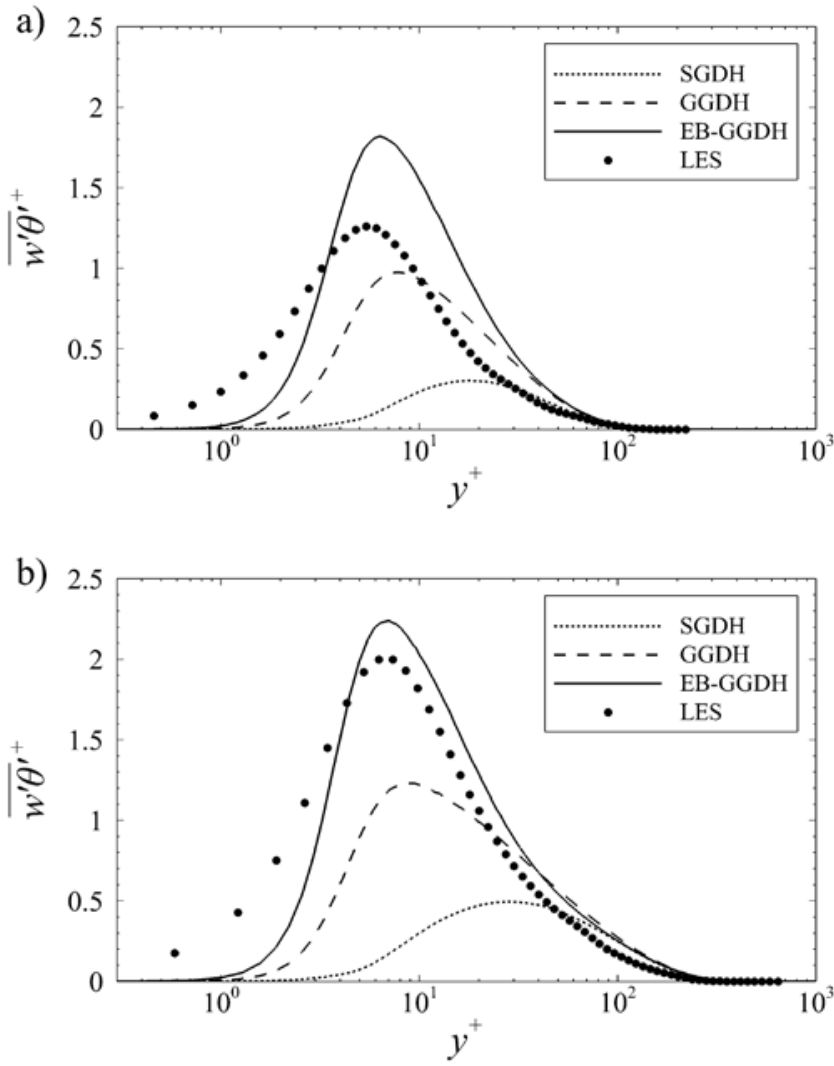

Fig. 8 - Span-wise turbulent heat flux. (a) Case 1; (b) Case 2. 

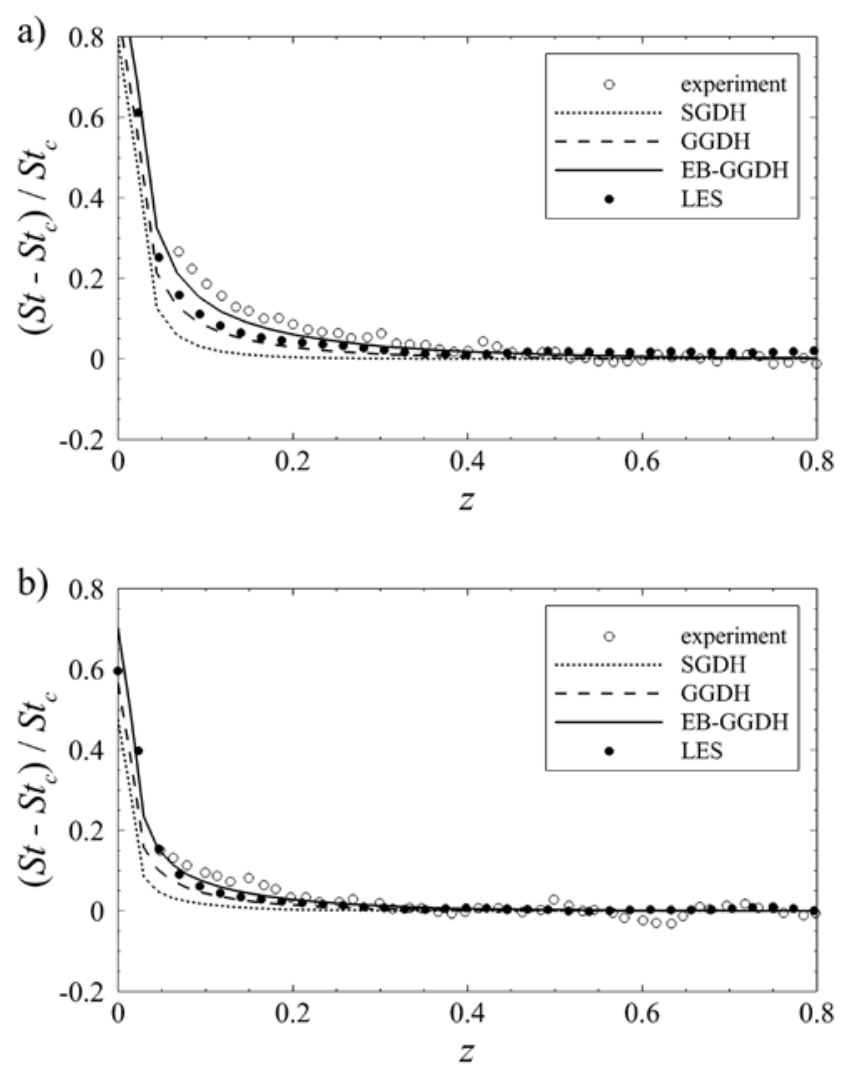

Fig. 9 - Relative increase of the Stanton number at the lateral edge. (a) Case 1; (b) Case 2.
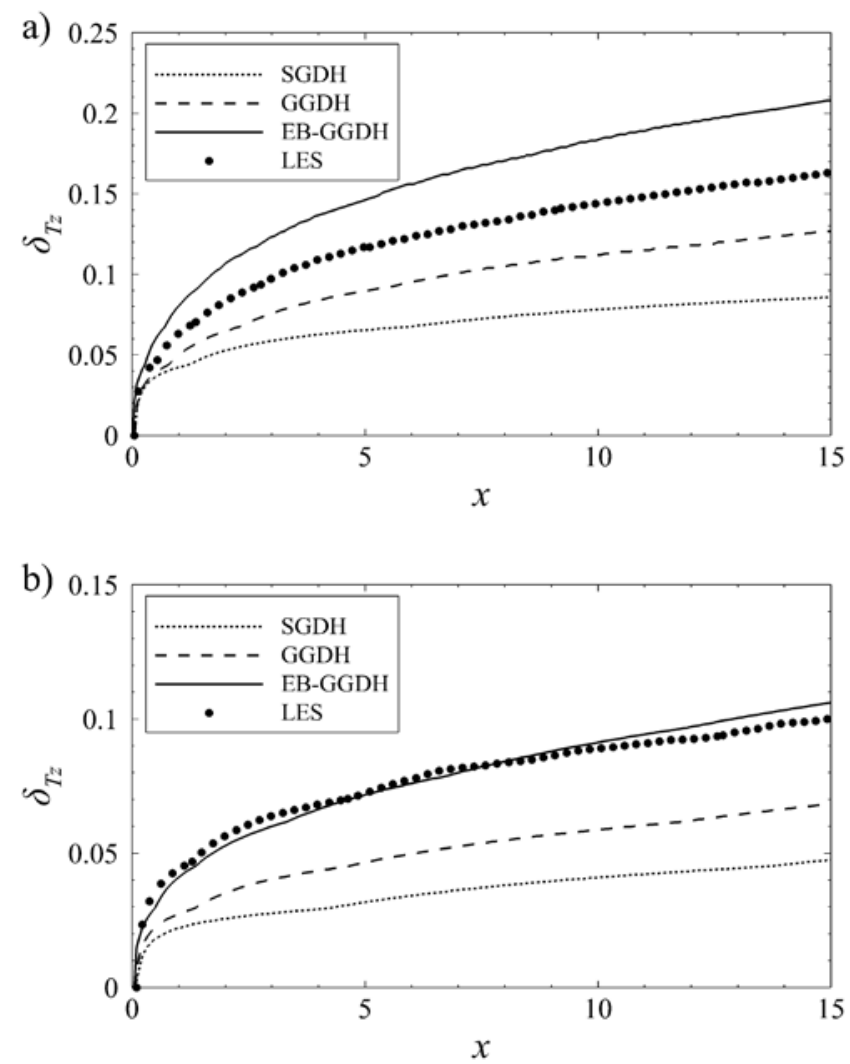

Fig. 10 - Span-wise temperature boundary layer thickness on the unheated plate (a) Case 1; (b) Case 2. 